\title{
Gas chromatography-time of flight/mass spectrometry-based metabonomics of changes in the urinary metabolic profile in osteoarthritic rats
}

\author{
HUI JIANG ${ }^{1,2}$, JIAN LIU ${ }^{3}$, XIU-JUAN QIN ${ }^{4}$, YUAN-YUAN CHEN ${ }^{1}$, \\ JIA-RONG GAO ${ }^{1}$, MEI MENG ${ }^{1}$, YUAN WANG ${ }^{3}$ and TING WANG ${ }^{4}$
}

\begin{abstract}
${ }^{1}$ Department of Pharmacy, The First Affiliated Hospital of Anhui University of Chinese Medicine, Hefei, Anhui 230031; ${ }^{2}$ College of Basic Medicine, Anhui Medical University, Hefei, Anhui 230032; ${ }^{3}$ Department of Rheumatism and Immunology, The First Affiliated Hospital of Anhui University of Chinese Medicine, Hefei, Anhui 230031; ${ }^{4}$ College of Pharmacy, Anhui University of Chinese Medicine, Hefei, Anhui 230038, P.R. China
\end{abstract}

Received November 19, 2015; Accepted December 12, 2016

DOI: $10.3892 / \mathrm{etm} .2018 .5788$

\begin{abstract}
The aim of the present study was to explore changes in the urinary metabolic spectrum in rats with knee osteoarthritis, using gas chromatography-time of flight/mass spectrometry (GC-TOF/MS) to determine the metabonomic disease pathogenesis. Sprague-Dawley rats were randomly divided into the control and model groups ( $\mathrm{n}=8 /$ group), and $20 \mu \mathrm{l}$ of $4 \%$ papain and $0.03 \mathrm{M} \mathrm{L}$-cysteine was injected into the right knee on days 1, 3 and 7 to establish the knee osteoarthritis model. Following 14 days, urine was collected over $12 \mathrm{~h}$ and cartilage ultrastructural damage was assessed by hematoxylin-eosin staining. GC-TOF/MS, combined with principal component analysis, partial least squares discriminant modeling and orthogonal partial least squares discriminant modeling, was used to analyze the changes in the metabolic spectrum trajectory and to identify potential biomarkers and their related metabolic pathways. Compared
\end{abstract}

Correspondence to: Professor Jian Liu, Department of Rheumatism and Immunology, The First Affiliated Hospital of Anhui University of Chinese Medicine, 117 Meishan Road, Hefei, Anhui 230031, P.R. China

E-mail: jhanbing@163.com

Abbreviations: GC-TOF/MS, gas chromatography-time of flight/mass spectrometry; SD, Sprague-Dawley; OA, osteoarthritis; KOA, knee osteoarthritis; TMCS, tri methyl chloro silane; IP, intraperitoneal perfusion; H\&E, hematoxylin and eosin; TEM, transmission electron microscopy; PCA, principal component analysis; PLS-DA, partial least squares discriminant analysis; OPLS-DA, orthogonal partial least-squares discriminant analysis; DEMO, demonstration; TIC, total ion current; VIP, variable importance projection; TCA, triglycerides; ROS, reactive oxygen species

Key words: knee osteoarthritis, urine, metabolomics, gas chromatography-time of flight/mass spectrometry, pathogenesis with the control group, the synovial cell lining of the knee joint exhibited proliferation, inflammatory cell infiltration and collagen fiber hyperplasia in the knee osteoarthritis group. A total of 23 potential biomarkers were identified, including alanine, $\alpha$-ketoglutarate, asparagine, maltose and glutamine. Furthermore, metabolomic pathogenesis of osteoarthritis may be related to disorders of amino acid metabolism, energy metabolism, fatty acid metabolism, vitamin $\mathrm{B}_{6}$ metabolism and nucleic acid metabolism.

\section{Introduction}

Osteoarthritis (OA) is a debilitating and multifactorial degenerative joint disease characterized by articular cartilage erosion and destruction, inflammation of the synovium, osteoproliferation of the joint edges, synovial hyperplasia and osteophyte formation at the joint margins (1). OA is associated with a loss of function of multiple diarthrodial joints in the body. Additionally, the related articular cartilage fibrosis, chaps, anabrosis and joint dislocation are major factors contributing to disability in the older population $(2,3)$. Although various studies have examined the molecular mechanisms of OA, alterations in the process of endogenous urinary metabolite formation during OA have not yet been published from the perspective of metabolomics $(4,5)$.

The discipline of metabolomics comprehensively examines changes in an organism's response to external processes under physiological and pathological conditions. This is achieved by observing how metabolite trajectories change over time when the biological system is stimulated in order to analyze differences in the metabolic fingerprint in different states $(6,7)$. Several different types of analytic platforms are able to achieve reasonable coverage of the metabolome, including nuclear magnetic resonance, Fourier transform infrared spectroscopy, high- performance liquid chromatography/mass spectrometry and gas chromatography-time of flight/mass spectrometry (GC-TOF/MS) (8). Among these, GC-TOF/MS is one of the most powerful and simple analytical platforms for achieving a comprehensive metabonomic analysis, with 
high sensitivity and reproducibility (9). Urine is commonly used as the analysis sample for metabolomics as GC-TOF/MS detection of urinary metabolomics has many advantages, such as being non-invasive, sensitive and rapid (10). Moreover, urinary metabonomic analysis is effective for monitoring the dynamic changes that occur during the progression of $\mathrm{OA}$. Therefore, metabolomic methods 'import' the information from OA pathogenesis research to assess potential biomarkers that show disease-specific changes that may be related to the physiological state of biological systems, which can be applied widely (11).

The aim of the present study was to determine changes in the urinary metabolic profile in rats with knee osteoarthritis using GC-TOF/MS techniques to assess the perturbed metabolites to elucidate the potential metabonomic pathogenesis of OA.

\section{Materials and methods}

Ethics statement. A total of 16 male Sprague-Dawley rats

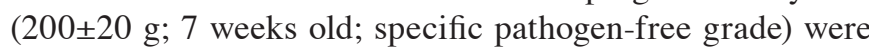
purchased from the Experimental Animal Center of Anhui Medical University (Anhui, China). The experimental protocol was approved by the Committee on the Ethics of Animal Experiments of Anhui University of Chinese Medicine (Anhui, China; permit number: 2012AH-038-01). All surgery procedures were performed under sodium pentobarbital anesthesia $(2 \mathrm{ml} / \mathrm{kg}$ ) and all efforts were made to minimize suffering.

Animal experimentation and sample collection. A total of 16 rats were allowed free access to food and water and were housed individually in a facility at $18-22^{\circ} \mathrm{C}$ with $40-60 \%$ humidity and a $12 \mathrm{~h}$ light/dark cycle. Following acclimatization for one week, rats were randomly divided into the control $(n=8)$ and knee osteoarthritis (KOA) model $(n=8)$ groups. KOA group rats were injected with $20 \mu \mathrm{l}$ of $4 \%$ papain and $0.03 \mathrm{M}$ L-cysteine into the right knee on days 1,3 and 7 to establish the rat models of KOA. Following 14 days, urine samples were collected over $12 \mathrm{~h}$ from all rats and stored at $-80^{\circ} \mathrm{C}$ until analysis.

Drugs and reagents. Papain, L-cysteine and urease from Canavalia ensiformis (Jack bean) were purchased from Sigma-Aldrich (Merck KGaA, Darmstadt, Germany). L-2-chlorobenzene alanine (103616-89-3) was purchased from Hemboug Biotechnology, Co., Ltd., (Shanghai, China) and N,O-Bis(trimethylsilyl)trifluoroacetamide, including 1\% v/v trimethylchlorosilane (TCMS) was purchased from REGIS Technologies, Inc., (Morton Grove, IL, USA).

Instruments for analysis. Gas chromatography chromatograph (Agilent 7890A; Agilent Technologies, Inc., Santa Clara, CA, USA); mass spectrometer (Leco Chroma TOF Pegasus 4D; Leco, Co., St Joseph, MI, USA); and $-80^{\circ} \mathrm{C}$ Ultra-Low Temperature Freezer (Thermo Fisher Scientific, Inc., Waltham, MA, USA).

Histopathology. Rats were anesthetized intraperitoneally with sodium pentobarbital $(2 \mathrm{ml} / \mathrm{kg}$; intraperitoneal perfusion, Sigma-Aldrich; Merck KGaA). Blood was removed from the abdominal aorta to sacrifice the rats. Knee joints of each rat was fixed in $10 \%$ neutral formalin for $24 \mathrm{~h}$ at room temperature, then embedded in paraffin and cut into $4 \mu \mathrm{m}$ sections. Paraffin-embedded liver sections were deparaffinized with xylene and rehydrated using an ethanol gradient (100-70\% v/v). The sections were then stained with $0.5 \%$ (w/v) hematoxylin (5-10 $\mathrm{min})$ and eosin (1-2 $\mathrm{min})$ at room temperature for general observation. Cartilage specimens were fixed in $2.5 \%$ glutaraldehyde stationary liquid for $2 \mathrm{~h}$ at room temperature, dehydrated and embedded in an epoxy resin. Samples were then baked for 12 and $1 \mathrm{~h}$ in an oven at $45^{\circ} \mathrm{C}$ and $65^{\circ} \mathrm{C}$, respectively. Ultra-thin $(70 \mathrm{~nm})$ sections were stained with toluidine blue for $30 \mathrm{~min}$ at room temperature. Finally, the sections were examined using a transmission electron microscope (JEM-1200X; JEOL Ltd., Tokyo, Japan).

Sample preparation. A total of $100 \mu \mathrm{l}$ from each sample was added to $1.5 \mathrm{ml}$ Eppendorf tubes and combined with $10 \mu \mathrm{l}$ of urease suspension $(160 \mathrm{mg} / \mathrm{ml}$ in water). Samples were vortexed for $10 \mathrm{sec}$ and subsequently incubated at $37^{\circ} \mathrm{C}$ for $1 \mathrm{~h}$ to decompose and remove excess urea, prior the addition of $0.35 \mathrm{ml}$ of the extraction liquid $\left(\mathrm{V}_{\text {methanol }}: \mathrm{V}_{\text {chloroform }}, 3: 1\right)$. L-2-chlorophenylalanine $(50 \mu \mathrm{l})$ from $0.2 \mathrm{mg} / \mathrm{ml}$ stock in $\mathrm{dH}_{2} \mathrm{O}$ was added to act as an internal standard. All samples were vortexed for a further $10 \mathrm{sec}$. Samples were centrifuged for $10 \mathrm{~min}$ at $4,200 \mathrm{x} \mathrm{g}$ at $4^{\circ} \mathrm{C}$. Supernatant was transferred $(\sim 0.35 \mathrm{ml})$ into $2-\mathrm{ml} \mathrm{GC}-\mathrm{TOF} / \mathrm{MS}$ glass vials. Extracts were dried in a vacuum concentrator without heating and $80 \mu \mathrm{l}$ methoxymethyl amine salt (dissolved in pyridine; final concentration, $20 \mathrm{mg} / \mathrm{ml}$ ) was added into dried metabolites, prior to incubation at $37^{\circ} \mathrm{C}$ for $2 \mathrm{~h}$ in an oven after mixing and sealing. Subsequently, $100 \mu \mathrm{l}$ N,O-Bis(trimethylsilyl)trifluoroacetamide (containing 1\% TCMS; v/v) was added into each sample before sealing and incubation at $70^{\circ} \mathrm{C}$ for $1 \mathrm{~h}$. A total of $10 \mu \mathrm{l}$ standard mixture, composed of fatty acid methyl esters (C8-C16, $1 \mathrm{mg} / \mathrm{ml}$; C18-C30, $0.5 \mathrm{mg} / \mathrm{ml}$ in chloroform) was combined with the sample and cooled to room temperature. Samples were thoroughly mixed prior to GC-TOF/MS analysis.

GC-TOF/MS analysis. An Agilent 7890 gas chromatograph system, coupled with a Pegasus high throughput time-of-flight mass spectrometer, was used for GC/TOF MS analysis. A DB-5MS capillary column, coated with 5\% diphenyl cross-linked with $95 \%$ dimethyl polysiloxane (inner diameter, $30 \mathrm{~m}$ x $250 \mu \mathrm{m}$; film thickness, $0.25 \mu \mathrm{m}$; J\&W Scientific Inc., Folsom, CA, USA) was used. A $1-\mu 1$ aliquot of the analyte was injected in splitless mode. The carrier gas utilized was helium and the front inlet purge flow was $3 \mathrm{ml} / \mathrm{min}$. There was agas flow rate of $20 \mathrm{ml} / \mathrm{min}$ through the column and the initial temperature was maintained at $50^{\circ} \mathrm{C}$ for $1 \mathrm{~min}$, which was raised to $330^{\circ} \mathrm{C}$ at a rate of $10^{\circ} \mathrm{C} / \mathrm{min}^{-1}$ and subsequently maintained at $330^{\circ} \mathrm{C}$ for $5 \mathrm{~min}$. The temperatures of injection, transfer line and ion source were 280,280 and $220^{\circ} \mathrm{C}$, respectively, and the electron impact mode consisted of $-70 \mathrm{eV}$ of energy. Full-scan mode, with an $\mathrm{m} / \mathrm{z}$ range of $85-600$, a rate of 20 spectra per second and a solvent delay of $366 \mathrm{sec}$, allowed mass spectrometry data to be obtained.

Statistical analysis. Raw peaks extraction, data baseline filtering and calibration of the baseline, peak alignment, 


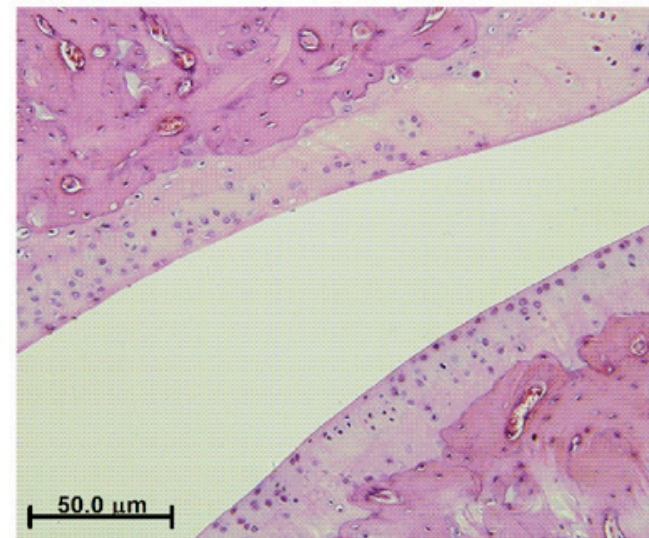

A

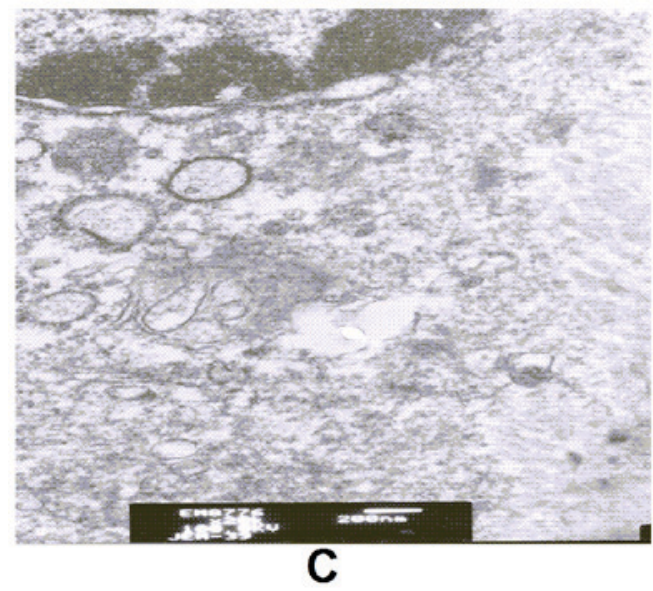

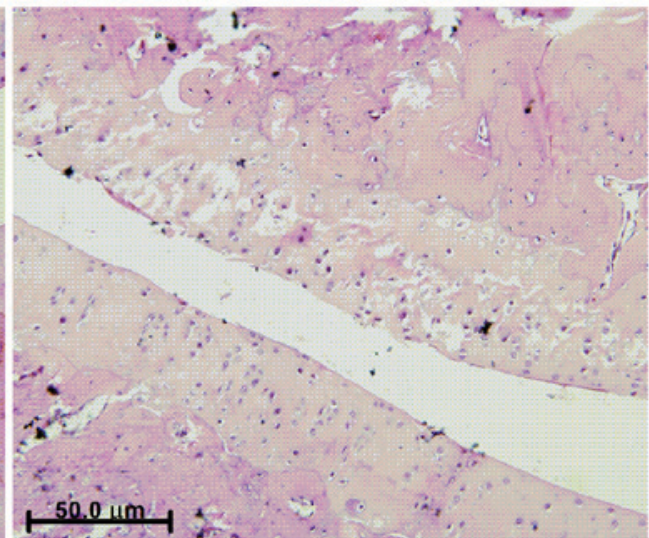

B

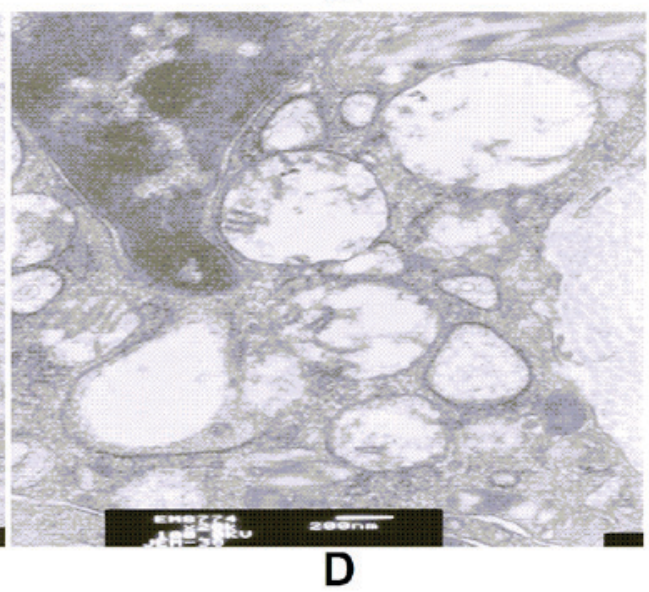

Figure 1. Histological examination of knee cartilage on knee osteoarthritis rats using H\&E staining (magnification, x400) and electron microscopy (magnification, x20,000). (A and B) H\&E staining. (C and D) Electron microscope. A and C: control group; B and D: model group. H\&E, hematoxylin and eosin.

deconvolution analysis, peak identification and integrated analysis were all performed using Chroma TOF4.3X software and Fiehn Rtx-5 database (both Laboratory Equipment Corp., Hayward, USA) (12). Principal component analysis (PCA), partial least squares discriminant analysis (PLS-DA) and orthogonal partial least-squares discriminant analysis (OPLS-DA) were utilized to analyze the resulting data, using demonstration Simca Software (V13.0, Umetrics; Satorius Stedim Biotech, Umea, Sweden). Statistical analysis was performed using one-way analysis of variance and Student-Newman-Keul's testing, using SPSS 17.0 software (SPSS, Inc., Chicago, IL, USA). P $<0.05$ was considered to indicate a statistically significant difference.

\section{Results}

Histological changes. In the knee joints of the control group, H\&E staining exhibited an orderly monolayer of synovial lining cells and cartilage, with an intact tidemark, normal cartilage cells, and no inflammatory cell infiltration or collagen fiber hyperplasia. In contrast, the joints in the KOA group indicated clear hyperplasia of the synovial lining cells, which was increased to three or four layers and gradual inflammatory cell infiltration and collagen fiber hyperplasia was observed.

Tissue ultrastructure in the control group showed that the cartilage cells were regularly arranged, with uniformly distributed central matrix fibers. Synovial cells exhibited a decreased number of rough endoplasmic reticulum and mitochondria in their cytoplasm, with microvilli protruding from the cell surface. In the KOA group, cartilage cells were irregularly shaped with a sparse matrix of plain fibers and synovial cells contained an increased number of rough endoplasmic reticulum in their cytoplasm. Furthermore, synovial cells exhibited mitochondrial degeneration with the appearance of some cavitation, as well as fewer microvilli protruding from the cell surface (Fig. 1).

Metabolite assignments with GC-TOF/MS spectroscopy. The total ion current (TIC) was expressed in response to all of the ions that contribute to the chromatogram, each peak of which represents a specific molecule (13). To investigate the differences between the metabolic spectra, the TIC of the metabolic spectra was analyzed. Chroma TOF4.3X software was used to correct the 1,144 data points by filling in blank entries, eliminating noise and subsequently centralizing to the interior labels. This resulted in the identification of 914 metabolic compounds with several distinct differences between the control and KOA spectra (Fig. 2).

$P C A$. PCA is a method to reduce the dimensionality of a dataset. Multiple variation variables are reduced into a few principal components that describe the structure of the original dataset, where the principal components of each 


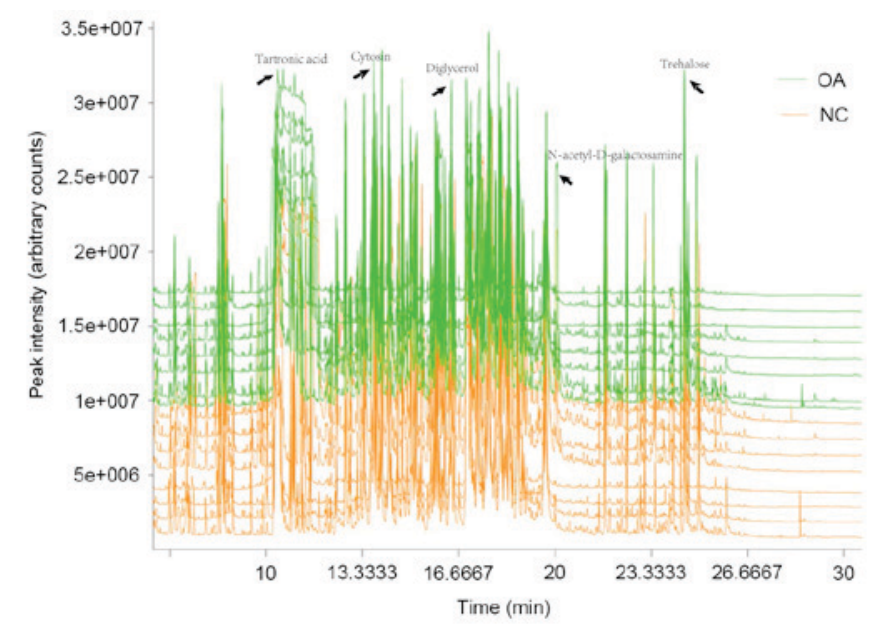

Figure 2. Gas chromatography-time of flight/mass spectrometry total ion current chromatograms (1144 peaks) of rat urine samples obtained from the control group and model groups. Each peak represents a specific molecule. $\mathrm{NC}$, control group; OA, osteoarthritis group.

sample represent the variations that best explain the entire sample dataset, which may be used to visually describe the changes in the metabolic patterns of the biological system in different physiological and pathological states $(14,15)$. In the present study, SIMCA-P ${ }^{+}$software was used to recognize and analyze the multivariate patterns in the centralized data. PCA was used for mean-centered scaling to visualize the data and present the distribution of samples. Control and KOA groups were not completely separated due to disturbances by various factors; therefore, large differences were detected between the groups (Fig. 3).

$P L S$-DA. To further separate the groups, PLS-DA was used. PLS-DA provides a deeper analysis of the main metabolic characteristics of biological samples in different states and in the present study, it identified the main variables that distinguish the sample principal component space, presenting the potential biomarkers that are characteristic of the different biological states (16). A PLS-DA text model, using leave-one-out cross validation with $\mathrm{R}^{2}$ and $\mathrm{Q}^{2}$ that represent the predictable variables and predictability of the model, were used to judge the validity of the model. The data indicated that the control and KOA groups were entirely separate after applying the PLS-DA $\left(\mathrm{R}^{2}=0.999 ; \mathrm{Q}^{2}=0.875\right)$, which revealed the stability, fit and predictivity of the mathematical model. Subsequently, the texting validity was assessed again by rearranging the experiment randomly through changing the sort order of the classification variable, $\mathrm{Y}$, and randomly assigning $\mathrm{Q}^{2}$ up to 200 times. Values of $\mathrm{R}^{2}=0.696$ and $Q^{2}=-0.293$ were obtained, which indicated that the model was reliable and not over-fit. The results of this analysis are shown in Fig. 4.

$O P L S-D A$. To further identify differences between the control and KOA groups, an orthogonal model was used and the first and second principal components based on OPLS-DA were analyzed (17). The two groups were separated completely after OPLS-DA (Fig. 5), which suggested that several potential biomarkers in the urine were clearly

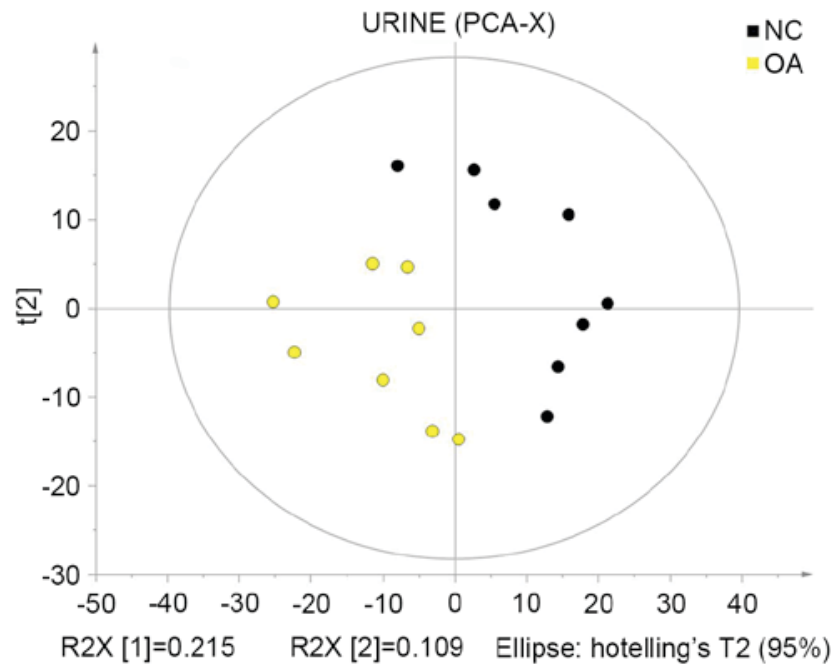

Figure 3. Principal component analysis score plot of rat urine samples obtained from the control and model groups. Control and knee osteoarthritis groups are not completely separated due to disturbances by various factors, thus large differences are indicated between the groups. NC, control group; OA, osteoarthritis group.

altered in the KOA group when compared with the control group. Loading plots revealed the distribution of variables and identified differences in the compound present in the samples or groups based on those variables (18). Each dot in the loading plot represented a metabolite and the dots near the center indicated smaller differences between the groups than those shown by dots far away from the center. The red dots indicated the 23 potential biomarkers identified in the present experiment.

Identification of endogenous metabolites. Significant differences between variables associated with their specificity as potential biomarkers were revealed, using variable importance projections of $>1$ with Student's t-tests $(\mathrm{P}<0.05$; Table I). The retention time was used to determine the mass spectrogram for each metabolite and the Fiehn Rtx-5 database was used to authenticate the general structure of the identified potential biomarkers (19). Based on that analysis, multiple components were revealed as potential biomarkers, including, alanine, $\alpha$-ketoglutarate, asparagine, maltose and glutamine, all of which were identified to be significantly altered in the KOA group when compared to the control group $(\mathrm{P}<0.05$; Table I).

Network analysis. To investigate potential biomarkers that may be involved in metabolic pathways related to OA and to visualize the connections between the various pathways, physiological, biochemical and pathophysiological data were combined with the Human Metabolome Database (http://www. hmdb.ca/about) and observed. Together, these relevant literatures, which were domestically and internationally resourced, provided quantitative and metabolic information on the organism metabolites. The identified potential biomarkers for KOA were revealed to predominantly be connected with amino acid metabolism, energy metabolism, nucleic acid metabolism, fatty acid metabolism and vitamin $\mathrm{B}_{6}$ metabolism (Fig. 6). 
A

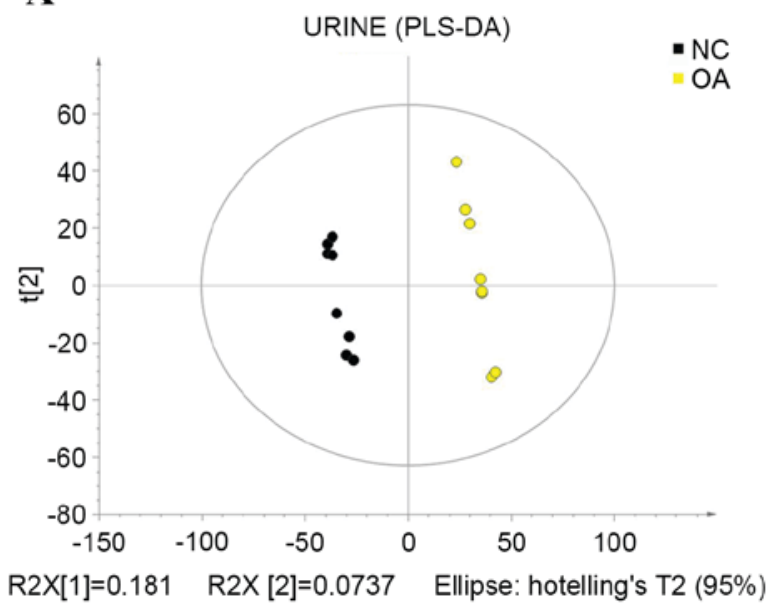

B

URINE.M22 (PLS-DA):validate model \$M22.DA (NC)intercepts:R2 $=(0.0,0.696)$, $\mathrm{Q} 2=(0.0,-0.293)$

$\cdot \mathrm{R} 2$

$\cdot \mathrm{Q} 2$

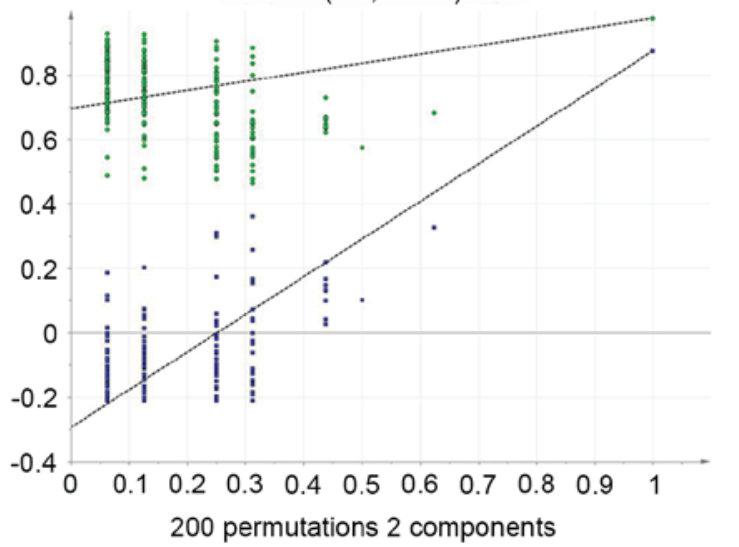

Figure 4. (A) Score plot of PLS-DA model was obtained from the control group and KOA model group and (B) two hundred permutations were performed, which were plotted according to the resulting $R^{2}$ and $Q^{2}$ values: $R^{2}=0.696$ and $Q^{2}=-0.293$. KOA, knee osteoarthritis; PLS-DA, partial least squares discriminant analysis; $\mathrm{NC}$, control group; OA, osteoarthritis group.
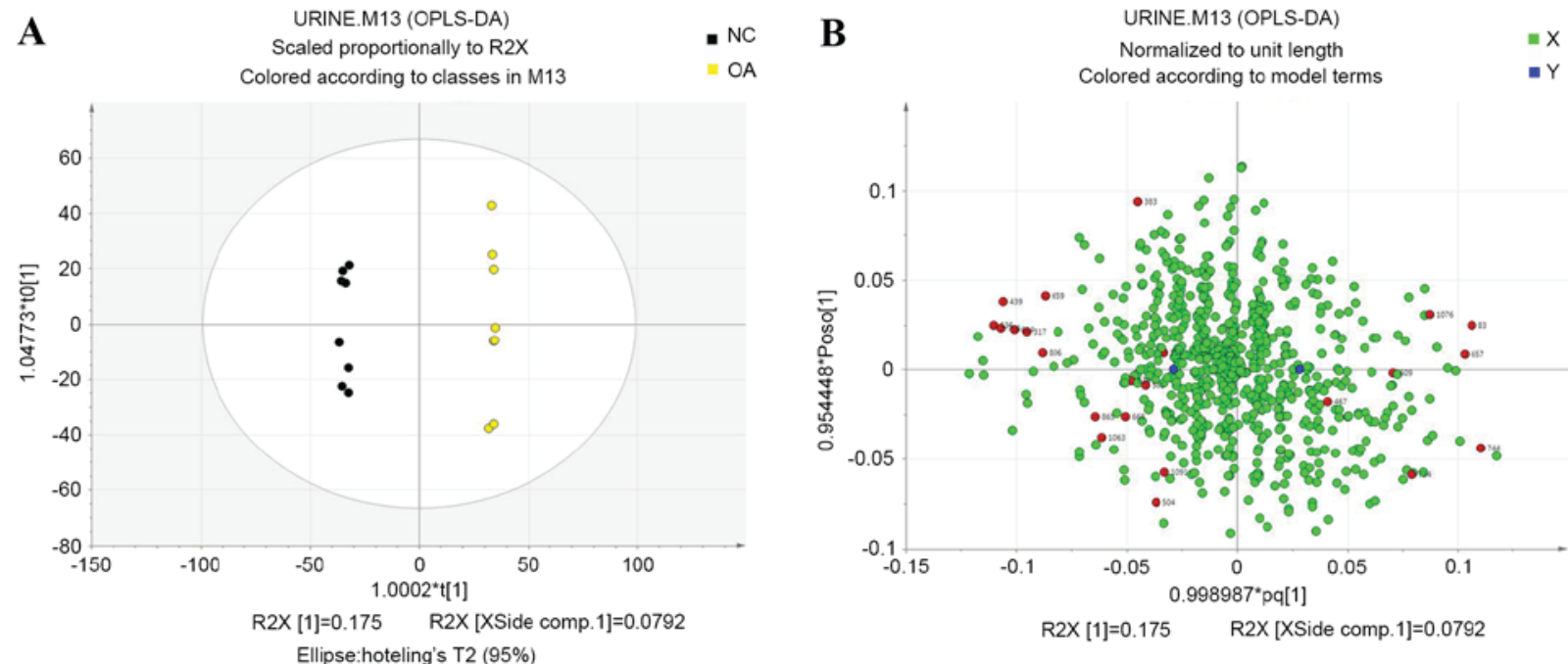

Figure 5. (A) Orthogonal projections to latent structures-discriminant analysis score plot of rat urine samples obtained from the control and knee osteoarthritis model groups. (B) Each dot in the loading plot represents a metabolic substance of the most influential variables, according to their respective contributions to the discrimination. Red dots indicated the potential biomarkers. OPLS-DA, orthogonal partial least-squares discriminant analysis; NC, control group; OA, osteoarthritis group.

\section{Discussion}

$\mathrm{OA}$ is a chronic and progressive joint disease that causes pathological characteristics, including articular cartilage erosion and destruction and synovial hyperplasia, particularly in the knee joint, which eventually results in the complete loss of joint function (20). Prior studies have indicated that injecting papain into the joint cavity is useful in constructing a KOA model, which identifies similar pathological alterations that are observed with OA in humans $(21,22)$. In addition, the KOA model may be established quickly, has a high success rate and is simple to perform, which explains why it is one of the classical models for investigating the pathogenesis of OA and drug screening $(23,24)$. The present study revealed that the synovial lining of the knee joint contained distinct collagen fiber hyperplasia, along with substantial inflammatory cell infiltration, which indicated the successful induction of KOA.

Genomics, proteomics and metabonomics together constitute 'systems biology', which is a discipline used to qualitatively and quantitatively analyze the small molecule metabolites of cells at specific times and under certain conditions, in order to describe the changes in the endogenous biological metabolites as a whole and their response to internal and external stimuli $(25,26)$. In the present study, GC-TOF/MS was used from the perspective of metabonomics to explore the pathogenesis of OA. A total of 23 potential biomarkers were identified, including alanine, $\alpha$-ketoglutarate, asparagine, maltose and glutamine, all of which may be correlated to amino acid metabolism, energy metabolism, fatty acid metabolism, vitamin $\mathrm{B}_{6}$ metabolism or nucleic acid metabolism (27-29). 
Table I. Biomarkers and change trends of KOA rats in each group.

\begin{tabular}{rrlrrr}
\hline No. & VarID & \multicolumn{1}{c}{ Possible compounds } & RT & VIP & P-value $^{\mathrm{a}}$ \\
\hline 1 & 83 & Alanine & 7.9005 & 3.1979 & 0.0099 \\
2 & 317 & Tartronic acid & 11.8798 & 2.8602 & 0.0061 \\
3 & 383 & Aminomalonic acid & 12.9363 & 1.3812 & 0.0435 \\
4 & 439 & Cytosin & 13.7735 & 3.2025 & 0.0078 \\
5 & 467 & $\alpha$-ketoglutaric acid & 14.2475 & 1.2320 & 0.0256 \\
6 & 504 & Asparagine & 14.7184 & 1.1209 & 0.0340 \\
7 & 516 & 5-aminovaleric acid & 14.8493 & 1.0065 & 0.0218 \\
8 & 535 & Xylose & 15.1326 & 3.3137 & 0.0007 \\
9 & 581 & Glutamine & 15.8341 & 1.2421 & 0.0094 \\
10 & 609 & Diglycerol & 16.3407 & 2.1399 & 0.0181 \\
11 & 614 & D-(glycerol 1-phosphate) & 16.3322 & 3.0259 & 0.0054 \\
12 & 639 & Shikimic acid & 16.7887 & 1.4643 & 0.0383 \\
13 & 657 & Homogentisic acid & 17.1321 & 3.1284 & 0.0038 \\
14 & 659 & N-carbamylglutamate & 17.1346 & 2.6190 & 0.0063 \\
15 & 668 & 2,8-dihydroxyquinoline & 17.2756 & 1.5259 & 0.0256 \\
16 & 734 & 4-pyridoxic acid & 18.1958 & 2.3907 & 0.0072 \\
17 & 744 & D-galacturonic acid & 18.4139 & 3.3468 & 0.0001 \\
18 & 776 & Urocanic acid & 18.9242 & 3.2128 & 0.0027 \\
19 & 806 & N-acetyl-D-galactosamine & 19.3819 & 2.6484 & 0.0021 \\
20 & 865 & 1,4-dihydroxy-2-naphthoic acid & 20.4740 & 1.9413 & 0.0111 \\
21 & 1063 & Trehalose & 24.8046 & 1.8507 & 0.0059 \\
22 & 1076 & Maltose & 25.0642 & 2.6504 & 0.0002 \\
23 & 1091 & Gentiobiose & 25.4649 & 1.0073 & 0.0052 \\
\hline
\end{tabular}

Screening of 1,166 peaks corresponding to the number of substances in the serum after VarID was used to import the software. ${ }^{a}$ Model group vs. control group. RT, retention time of the substance; KOA, knee osteoarthritis; VIP, variable importance projection.

Glutamine is a non-essential amino acid that is found in abundance throughout the body and is involved in multiple metabolic processes. It is synthesized from glutamic acid and ammonia (30). N-carbamylglutamate is an azyl product of glutamate through carbamylation. Urocanic acid is a deamination (breakdown) product of histidine that is related to oxidative damage as a direct scavenger of reactive oxygen species (ROS) (31). 5-aminovaleric acid is a type of ornithine that participates in the urea cycle through amino acid catabiosis and by offering an amidogen to the urea cycle (32). Asparagine is generated from oxaloacetic acid by deamination, which then enters the tricarboxylic acid (TCA) cycle (33). Previous studies have revealed that hydroxyl radicals, $\cdot \mathrm{OH}$, are important $\mathrm{ROS}$ in $\mathrm{OA}$, which possess the fastest reactive chemical properties (34-35). Hydroxyl radicals may react with all types of amino acids, disrupting the ordered structure that produces amino acid chains of proteins and inducing collagen degradation and destruction of articular joint function in OA (36). In the present study, the contents of glutamine, $\mathrm{N}$-carbamylglutamate, urocanic acid, 5-aminovaleric acid and asparagine were clearly decreased in the urine samples from the KOA group of rats, compared with that of the control group, which may be related to the generation of $\cdot \mathrm{OH}$ as this disrupts the structure of amino acids.
Alanine is one of the 20 different types of amino acids that constitute the proteins of the human body. Through the alanine-glucose cycle, alanine participates in the lactic acid-glucose cycle and subsequently enters the TCA cycle to regulate energy metabolism (37). In cases of chronic inflammation, such as joint inflammation in OA, lactic acid is a characteristic substance for detection that is also a pathogenic factor in the transformation of cells and development of autoantigenicity. When the lactic acid concentration becomes elevated an organism enters acidosis, thus further inducing the development of inflammation (38). In the present study, increased alanine content in the KOA group suggested an abnormal amino acid metabolism occurring in those animals.

Inflammatory factors in OA inhibit activation of insulin signaling, glycolysis and the pentose phosphate pathway, thereby increasing blood glucose levels (39). Phosphoenolpyruvate in the glycolysis pathway and erythrose 4-phosphate in the pentose phosphate pathway are synthesis products of shikimic acid that may be broken down into chorismic acid to generate 1,4-dihydroxy-naphthoic acid (40). N-acetyl-D-galactosamine, trehalose, gentiobiose and xylose provide energy for the organism through the pentose phosphate and glycolysis pathways $(41,42)$. Maltose is a primary disaccharide in the human diet, formed from two units of glucose joined with 


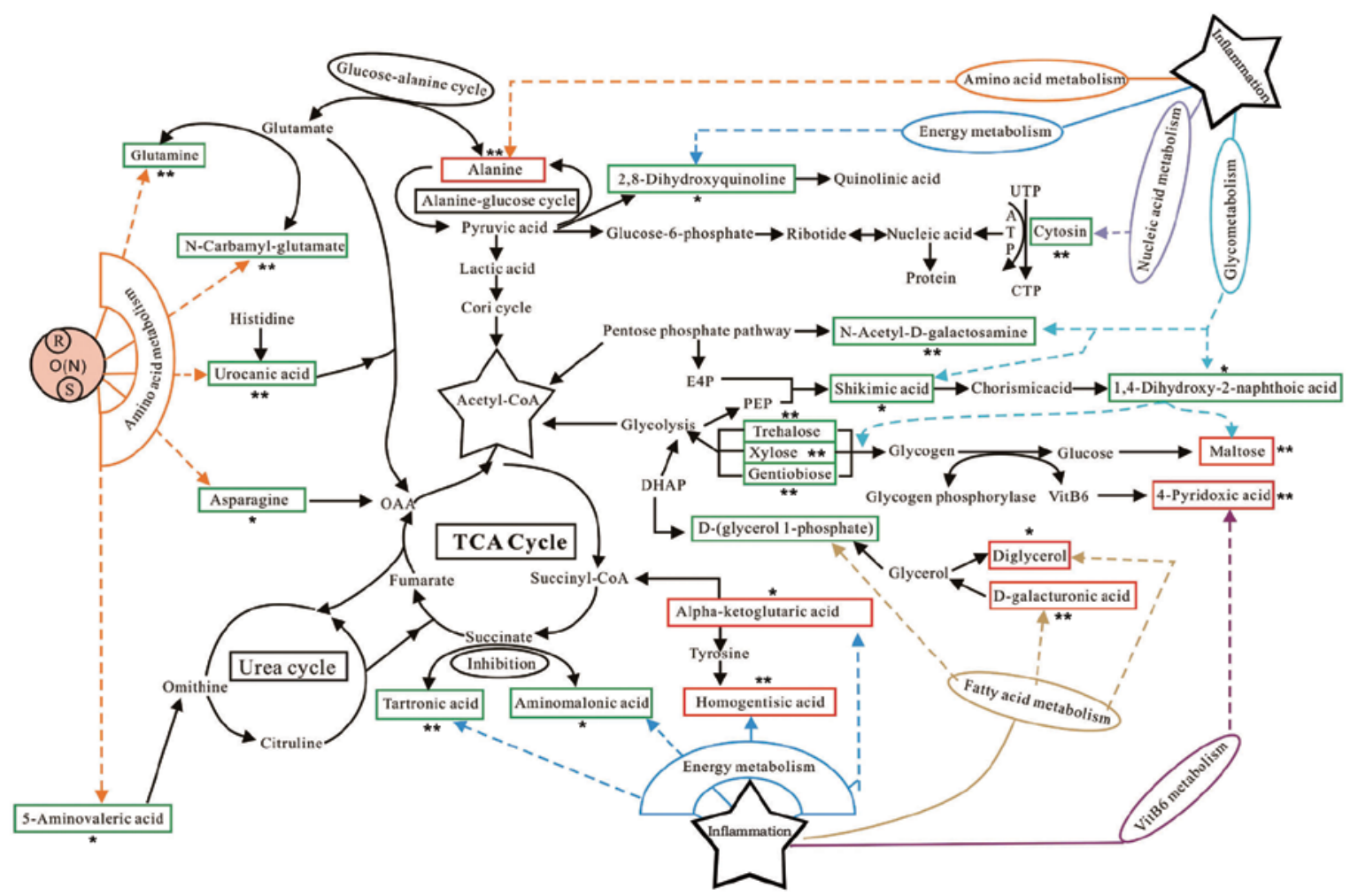

Figure 6. Pathway network analysis. Metabolites in the red frame depict those that are increased in the KOA model group when compared to the control group; metabolites in the green frame depict those that are decreased in the KOA model group when compared to the control group. Both denote the endogenous metabolites identified which are associated with various possible metabolic pathways. KOA, knee osteoarthritis; TCA, triglycerides; UTP, uridine-triphosphate; ATP, adenosine triphosphate; CTP, cytidine triphosphate; OAA, oxaloacetate; E4P, erythrose 4-phosphate; DMAP, N,N-Dimethylpyridin-4-amine; succinyl CoA, Succinyl-Coenzyme A; "P<0.05 vs. control, ${ }^{* *} \mathrm{P}<0.01$ vs. control.

an alpha linkage. It is the second member of an important biochemical series of glucose chains (43). In the present study, the contents of shikimic acid, 1,4-dihydroxy-naphthoic acid, $\mathrm{N}$-acetyl-D-galactosamine, trehalose, gentiobiose and xylose were decreased, while the maltose content was increased in the KOA group when compared with the control group, which may be related to the inhibition of the glycolysis and pentose phosphate pathways and increased blood glucose levels.

Tartronic acid and aminomalonic acid are two products of malonic acid, the structure of which is similar to succinic acid (44). Malonic acid is the archetypal example of a competitive inhibitor, which acts against succinate dehydrogenase (complex II) in the respiratory electron transport chain, thus mediating the TCA cycle (45). Oxidase activity was decreased in the present study during inflammatory damage caused by inflammatory factors and such incomplete oxidation may lead to lactic acid, pyruvic acid and $\alpha$-ketoglutaric acid accumulation in the inflammation center. Furthermore, $\alpha$-ketoglutaric acid may also be modified to succinyl-coenzyme A (succinyl CoA) through oxidation and decarboxylation. Succinyl-CoA produces further succinic acid and participates in the TCA cycle. Homogentisic acid is an intermediate of the metabolic breakdown of tyrosine and phenylalanine, which is found in the urine in cases of alkaptonuria (46). Mitochondrial dysfunction has been demonstrated in OA and the vast majority of enzymes that participate in TCA are located in the mitochondria of the cartilage cells, which may result in metabolic disturbance and abnormal metabolic intermediates from the TCA (47). The present study revealed that the content of $\alpha$-ketoglutaric acid and homogentisic acid were increased and tartronic acid and aminomalonic acid were decreased in the KOA group, suggesting a possible association with abnormal energy metabolism.

2,8-hydroxyquinoline is a product of pyruvate metabolism that is further broken down into quinolinic acid. Quinolinic acid is an anti-inflammatory factor that exhibits catabolic, anti-microbial, anti-tumor, anti-fungal and anti-cancer effects (48). Oxygen depletion is one of the major features of OA, which prevents 2,8-hydroxyquinoline from generating quinolinic acid, thus aggravating OA-related inflammation. This interpretation is consistent with the present results, where the content of 2,8-hydroxyquinoline was decreased in the KOA group when compared with the control group.

Glycerol is an important component of triglycerides (fats and oils) and phospholipids, which are generated by D-(glycerol-1-phosphate). The glycerol component may be converted into glucose by the liver to provide energy for cellular metabolism. D-(glycerol-1-phosphate) is one of the phosphoric acid derivatives of glycerol and is generated from dihydroxyacetone phosphate by glycerophosphate dehydrogenase during glycolysis. Inflammatory factors inhibit the activation of insulin signaling and the glycolysis pathway in OA, resulting in decreases of D-(glycerol-1-phosphate) $(49,50)$. However, fat mobilization is accelerated, leading to the induction and activation of phospholipase $\mathrm{C}$ during $\mathrm{OA}$, which functions as a secondary messenger. Glycerol formed 
from diglyceride also acts as a secondary messenger and levels of glycerol are increased during OA, further inducing the production of inflammatory factors (51). In the present study, the contents of diglycerol and D-(glycerol-1-phosphate) were increased in the KOA group when compared with the control, which may be associated with disorders of fatty acid metabolism.

4-pyridoxic acid is the catabolic product of vitamin $B_{6}$. Together with vitamin $\mathrm{B}_{6}$, glycogen phosphorylase accelerates the transformation of glycogen into glucose (52). Inflammatory factors during OA inhibit the activation of insulin signaling, increasing blood glucose levels. In the present study, 4-pyridoxic acid content was increased in the KOA group when compared with the control group. Increases in 4-pyridoxic acid content may be related to disorders in vitamin $\mathrm{B}_{6}$ metabolism.

Inflammation during OA and ROS can lead to degraded bases resulting from nucleic acids attacked by the free radicals that induce collagen degradation, which may subsequently destroy joint function (53). Cytosine is a pyrimidine base that is a fundamental unit of nucleic acids and is involved in the synthesis and metabolism of nucleic acids. Compared with the control group, the cytosine content was decreased in the KOA group, which may be related to disorders of nucleic acid metabolism.

In conclusion, the metabonomic pathogenesis of OA may be related to disorders of amino acid metabolism, energy metabolism, fatty acid metabolism, vitamin $\mathrm{B}_{6}$ metabolism and nucleic acid metabolism.

\section{Acknowledgements}

The present study was financially supported by the National Key Technology Support Program (grant no. 2012BAI26B03). We are grateful to Ms. Jie Xu and Dr Junliang Deng (Biotree Bio-technology Co., Ltd., Shanghai, China) for providing help with data analysis.

\section{Competing interests}

The authors declare that they have no competing interests.

\section{References}

1. Valdes AM and Spector TD: The clinical relevance of genetic susceptibility to osteoarthritis. Best Pract Res Clin Rheumatol 24: 3-14, 2010.

2. Adams SB Jr, Setton LA, Kensicki E, Bolognesi MP, Toth AP and Nettles DL: Global metabolic profiling of human osteoarthritic synovium. Osteoarthritis Cartilage 20: 64-67, 2012.

3. Puig-Junoy J and Ruiz Zamora A: Socio-economic costs of osteoarthritis: A systematic review of cost-of-illness studies. Semin Arthritis Rheum 44: 531-541, 2015.

4. Wei Y and Bai L: Recent advances in the understanding of molecular mechanisms of cartilage degeneration, synovitis and subchondral bone changes in osteoarthritis. Connect Tissue Res 57: 245-261, 2016.

5. Li H, Hao Z, Zhao L, Liu W, Han Y, Bai Y and Wang J: Comparison of molecular mechanisms of rheumatoid arthritis and osteoarthritis using gene microarrays. Mol Med Rep 13: 4599-4605, 2016.

6. Fujiwara Y, Kobayashi T, Chayahara N, Imamura Y, Toyoda M, Kiyota N, Mukohara T, Nishiumi S, Azuma T, Yoshida M and Minami H: Metabolomics evaluation of serum markers for cachexia and their intra-day variation in patients with advanced pancreatic cancer. PLoS One 9: e113259, 2014.
7. Sun H, Zhang AH, Zou DX, Sun WJ, Wu XH and Wang XJ: Metabolomics coupled with pattern recognition and pathway analysis on potential biomarkers in liver injury and hepatoprotective effects of yinchenhao. Appl Biochem Biotechnol 173: 857-869, 2014.

8. Yu K, Sheng G, Sheng J, Chen Y, Xu W, Liu X, Cao H, Qu H, Cheng Y and Li L: A metabonomic investigation on the biochemical perturbation in liver failure patients caused by hepatitis B virus. J Proteome Res 6: 2413-2419, 2007.

9. Ressom HW, Xiao JF, Tuli L, Varghese RS, Zhou B, Tsai TH, Ranjbar MR, Zhao Y, Wang J, Di Poto C, et al: Utilization of metabolomics to identify serum biomarkers for hepatocellular carcinoma in patients with liver cirrhosis. Anal Chim Acta 743: 90-100, 2012.

10. Wu D, Cai S, Chen M, Ye L, Chen Z, Zhang H, Dai F, Wu F and Zhang G: Tissue metabolic responses to salt stress in wild and cultivated barley. PLoS One 8: e55431, 2013.

11. Gou X, Tao Q, Feng Q, Peng J, Zhao Y, Dai J, Wang W, Zhang Y, $\mathrm{Hu} \mathrm{Y}$ and Liu P: Urine metabolic profile changes of CCl4-liver fibrosis in rats and intervention effects of Yi Guan Jian Decoction using metabonomic approach. BMC Complement Altern Med 13: $123,2013$.

12. Kind T, Wohlgemuth G, Lee DY, Lu Y, Palazoglu M, Shahbaz S and Fiehn O: FiehnLib: Mass spectral and retention index libraries for metabolomics based on quadrupole and time-of-flight gas chromatography/mass spectrometry. Anal Chem 81: 10038-10048, 2009.

13. Wu H, Liu T, Ma C, Xue R, Deng C, Zeng H and Shen X: GC/MS-based metabolomic approach to validate the role of urinary sarcosine and target biomarkers for human prostate cancer by microwave-assisted derivatization. Anal Bioanal Chem 401: 635-646, 2011.

14. Huang X, Shao L, Gong Y, Mao Y, Liu C, Qu H and Cheng Y: A metabonomic characterization of $\mathrm{CCl} 4$-induced acute liver failure using partial least square regression based on the GC/MS metabolic profiles of plasma in mice. J Chromatogr B Analyt Technol Biomed Life Sci 870: 178-185, 2008.

15. Rachakonda V, Gabbert C, Raina A, Bell LN, Cooper S, Malik S and Behari J: Serum metabolomic profiling in acute alcoholic hepatitis identifies multiple dysregulated pathways. PLoS One 9: e113860, 2014.

16. Li H, Xia X, Li X, Naren G, Fu Q, Wang Y, Wu C, Ding S, Zhang S, Jiang $\mathrm{H}$, et al: Untargeted metabolomic profiling of amphenicol-resistant Campylobacter jejuni by ultra-high-performance liquid chromatography-mass spectrometry. J Proteome Res 14: 1060-1068, 2015.

17. Jin H, Qiao F, Chen L, Lu C, Xu L and Gao X: Serum metabolomic signatures of lymph node metastasis of esophageal squamous cell carcinoma. J Proteome Res 13: 4091-4103, 2014.

18. Ji P, Wei Y, Sun H, Xue W, Hua Y, Li P, Zhang W, Zhang L, Zhao $\mathrm{H}$ and Li J: Metabolomics research on the hepatoprotective effect of Angelica sinensis polysaccharides through gas chromatography-mass spectrometry. J Chromatogr B Analyt Technol Biomed Life Sci 973C: 45-54, 2014.

19. Wang L, Hou E, Wang Y, Wang Y, Yang L, Zheng X, Xie G, Sun $\mathrm{Q}$, Liang $\mathrm{M}$ and Tian Z: Reconstruction and analysis of correlation networks based on GC-MS metabolomics data for young hypertensive men. Anal Chim Acta 854: 95-105, 2015.

20. Stannus O, Jones G, Cicuttini F, Parameswaran V, Quinn S, Burgess J and Ding C: Circulating levels of IL-6 and TNF-alpha are associated with knee radiographic osteoarthritis and knee cartilage loss in older adults. Osteoarthritis Cartilage 18: 1441-1447, 2010.

21. Li X, Lang W, Ye H, Yu F, Li H, Chen J, Cai L, Chen W, Lin R, Huang Y and Liu X: TouguXiaotong capsule inhibits the tidemark replication and cartilage degradation of papain-induced osteoarthritis by the regulation of chondrocyte autophagy. Int $\mathrm{J}$ Mol Med 31: 1349-1356, 2013.

22. Guo CQ, Ji B, Chen YN, Zhong DW, Jin Y, Liu QG, Guo MW, Zhang Y, Liu NG and Chen ZL: Affection of acupotomy lysis on leu-enkephalin (L-ENK) content in different parts of centrum of rats with knee osteoarthritis. Zhongguo Gu Shang 24: 656-658, 2011 (In Chinese).

23. Aigner T, Cook JL, Gerwin N, Glasson SS, Laverty S, Little CB, McIlwraith W and Kraus VB: Histopathology atlas of animal model systems-overview of guiding principles. Osteoarthritis Cartilage 18 (Suppl 3): S2-S6, 2010.

24. Pritzker KP: Animal models for osteoarthritis: Processes, problems and prospects. Ann Rheum Dis 53: 406-420, 1994. 
25. He J, Chen J, Wu L, Li G and Xie P: Metabolic response to oral microcystin-LR exposure in the rat by NMR-based metabonomic study. J Proteome Res 11: 5934-5946, 2012.

26. Zira A, Kostidis S, Theocharis S, Sigala F, Engelsen SB, Andreadou I and Mikros E: 1H NMR-based metabonomics approach in a rat model of acute liver injury and regeneration induced by CCl4 administration. Toxicology 303: 115-124, 2013.

27. Neis EP, Sabrkhany S, Hundscheid I, Schellekens D, Lenaerts K, Olde Damink SW, Blaak EE, Dejong CH and Rensen SS: Human splanchnic amino-acid metabolism. Amino Acids 49: 161-172, 2017.

28. Klimaszewska-Łata J, Gul-Hinc S, Bielarczyk H, Ronowska A, Zyśk M, Grużewska K, Pawełczyk T and Szutowicz A: Differential effects of lipopolysaccharide on energy metabolism in murine microglial N9 and cholinergic SN56 neuronal cells. J Neurochem 133: 284-297, 2015.

29. Zuo H, Ueland PM, Eussen SJ, Tell GS, Vollset SE, Nygård O, Midttun $\varnothing$, Meyer K and Ulvik A: Markers of vitamin B6 status and metabolism as predictors of incident cancer: The Hordaland Health Study. Int J Cancer 136: 2932-2939, 2015

30. Shan L, Liao F, Jin H, Ye F, Tong P, Xiao L, Zhou J and Wu C: Plasma metabonomic profiling of lumbar disc herniation and its traditional Chinese medicine subtypes in patients by using gas chromatography coupled with mass spectrometry. Mol Biosyst 10: 2965-2973, 2014.

31. Jiang M, Chen T, Feng H, Zhang Y, Li L, Zhao A, Niu X, Liang F, Wang M, Zhan J, et al: Serum metabolic signatures of four types of human arthritis. J Proteome Res 12: 3769-3779, 2013.

32. Hiong KC, Loong AM, Chew SF and Ip YK: Increases in urea synthesis and the ornithine-urea cycle capacity in the giant African snail, Achatina fulica, during fasting or aestivation, or after the injection with ammonium chloride. J Exp Zool A Comp Exp Biol 303: 1040-1053, 2005.

33. Wang X, Liu Y, Li S, Pi D, Zhu H, Hou Y, Shi H and Leng W: Asparagine attenuates intestinal injury, improves energy status and inhibits AMP-activated protein kinase signalling pathways in weaned piglets challenged with Escherichia coli lipopolysaccharide. Br J Nutr 114: 553-565, 2015.

34. Wu L, Liu H, Li L, Liu H, Cheng Q, Li H and Huang H: Mitochondrial pathology in osteoarthritic chondrocytes. Curr Drug Targets 15: 710-719, 2014.

35. Lepetsos P and Papavassiliou AG: ROS/oxidative stress signaling in osteoarthritis. Biochim Biophys Acta 1862: 576-591, 2016.

36. Borel M, Pastoureau P, Papon J, Madelmont JC, Moins N, Maublant J and Miot-Noirault E: Longitudinal profiling of articular cartilage degradation in osteoarthritis by high-resolution magic angle spinning 1H NMR spectroscopy: Experimental study in the meniscectomized guinea pig model. J Proteome Res 8: 2594-2600, 2009.

37. Chen L, Luo Z, Fu W, Liao X, Cui Z and Zhou J: Detection of urinary metabolomics before and after Pringle maneuver-induced liver ischemia and reperfusion injury in rats using gas chromatography-mass spectrometry. Dis Markers 35: 345-351, 2013

38. Wu C, Lei R, Tiainen M, Wu S, Zhang Q, Pei F and Guo X: Disordered glycometabolism involved in pathogenesis of Kashin-Beck disease, an endemic osteoarthritis in China. Exp Cell Res 326: 240-250, 2014.

39. Mickiewicz B, Heard BJ, Chau JK, Chung M, Hart DA, Shrive NG, Frank CB and Vogel HJ: Metabolic profiling of synovial fluid in a unilateral ovine model of anterior cruciate ligament reconstruction of the knee suggests biomarkers for early osteoarthritis. J Orthop Res 33: 71-77, 2015.

40. Dolatshahi S, Fonseca LL and Voit EO: New insights into the complex regulation of the glycolytic pathway in Lactococcus lactis. II. Inference of the precisely timed control system regulating glycolysis. Mol Biosyst 12: 37-47, 2016.
41. Kogure T, Kubota T, Suda M, Hiraga K and Inui M: Metabolic engineering of Corynebacterium glutamicum for shikimate overproduction by growth-arrested cell reaction. Metab Eng 38: 204-216, 2016

42. Rossouw D, Heyns EH, Setati ME, Bosch S and Bauer FF. Adjustment of trehalose metabolism in wine Saccharomyces cerevisiae strains to modify ethanol yields. Appl Environ Microbiol 79: 5197-5207, 2013.

43. Mathiselvam M, Ramkumar V,Loganathan D and Pérez S: Effect of distal sugars and interglycosidic linkage on the $\mathrm{N}$-glycoprotein linkage region conformation: Synthesis and X-ray crystallographic investigation of $\beta-1-\mathrm{N}$-alkanamide derivatives of cellobiose and maltose as disaccharide analogs of the conserved chitobiosylasparagine linkage. Glycoconj J 31: 71-87, 2014.

44. Motta A, Paris D, D'Amato M, Melck D, Calabrese C, Vitale C, Stanziola AA, Corso G, Sofia M and Maniscalco M: NMR metabolomic analysis of exhaled breath condensate of asthmatic patients at two different temperatures. J Proteome Res 13: 6107-6120, 2014

45. Minato Y, Fassio SR and Häse CC: Malonate inhibits virulence gene expression in Vibrio cholerae. PLoS One 8: e63336, 2013.

46. Milan AM, Hughes AT, Davison AS, Devine J, Usher J, Curtis S, Khedr M, Gallagher JA and Ranganath LR: The effect of nitisinone on homogentisic acid and tyrosine: A two-year survey of patients attending the National Alkaptonuria Centre, Liverpool. Ann Clin Biochem 54: 323-330, 2017.

47. Henrotin Y, Kurz B and Aigner T: Oxygen and reactive oxygen species in cartilage degradation: Friends or foes? Osteoarthritis Cartilage 13: 643-654, 2005.

48. Qi Y, Li S, Pi Z, Song F, Lin N, Liu S and Liu Z: Metabonomic study of Wu-tou decoction in adjuvant-induced arthritis rat using ultra-performance liquid chromatography coupled with quadrupole time-of-flight mass spectrometry. J Chromatogr B Analyt Technol Biomed Life Sci 953-954: 11-19, 2014

49. Priori R, Scrivo R, Brandt J, Valerio M, Casadei L, Valesini G and Manetti C: Metabolomics in rheumatic diseases: The potential of an emerging methodology for improved patient diagnosis, prognosis, and treatment efficacy. Autoimmun Rev 12: 1022-1030, 2013

50. Wurtz P, Wang Q, Kangas AJ, Richmond RC, Skarp J, Tiainen M, Tynkkynen T, Soininen P, Havulinna AS, Kaakinen M, et al: Metabolic signatures of adiposity in young adults: Mendelian randomization analysis and effects of weight change. PLoS Med 11: e1001765, 2014

51. Zini N, Lisignoli G, Solimando L, Bavelloni A, Grassi F, Guidotti L, Trimarchi C, Facchini A and Maraldi NM: IL1-beta and TNF-alpha induce changes in the nuclear polyphosphoinositide signalling system in osteoblasts similar to that occurring in patients with rheumatoid arthritis: An immunochemical and immunocytochemical study. Histochem Cell Biol 120: 243-250, 2003.

52. Yue R, Zhao L, Hu Y, Jiang P, Wang S, Xiang L, Liu W, Zhang W and Liu R: Rapid-resolution liquid chromatography TOF-MS for urine metabolomic analysis of collagen-induced arthritis in rats and its applications. J Ethnopharmacol 145: 465-475, 2013.

53. Madsen RK, Lundstedt T, Gabrielsson J, Sennbro CJ, Alenius GM, Moritz T, Rantapää-Dahlqvist S and Trygg J: Diagnostic properties of metabolic perturbations in rheumatoid arthritis. Arthritis Res Ther 13: R19, 2011.

This work is licensed under a Creative Commons Attribution-NonCommercial-NoDerivatives 4.0 International (CC BY-NC-ND 4.0) License. 